\title{
Poligrafía pediátrica: Experiencia de 6 años
}

\section{Pediatric polygraphy: A 6-year experience}

\section{Daniel Zenteno $^{\mathrm{a}, \mathrm{b}}$, Daniela Verbal ${ }^{\mathrm{b}}$, Ximena Navarro $^{\mathrm{a}}$, Gerardo Torres ${ }^{\mathrm{a}, \mathrm{c}}$, Carla Rivas B. ${ }^{a}$, Iván Rodríguez-Núñez ${ }^{c}$, María José Elso ${ }^{\text {a,d }}$, Jaime Tapia ${ }^{\mathrm{a}}$}

\author{
aServicio de Pediatría, Hospital Guillermo Grant Benavente, Concepción, Chile \\ bDepartamento de Pediatría, Facultad de Medicina, Universidad de Concepción, Chile \\ 'Departamento de Kinesiología, Facultad de Medicina, Universidad de Concepción, Chile \\ dDepartamento de Especialidades Médicas, Facultad de Medicina, Universidad de Concepción
}

Recibido: 29 de mayo de 2018; aceptado: 14 de febrero de 2019

\begin{abstract}
Resumen
El diagnóstico precoz de los Trastornos Respiratorios del Sueño (TRS) puede permitir una intervención oportuna. La poligrafía (PG) es una alternativa confiable y accesible en la actualidad. Objetivo: Describir y analizar PG de niños $\geq 1$ año con sospecha de TRS. Pacientes y Método: Se incluyeron PG de niños y adolescentes $\geq 1$ año de edad con sospecha de TRS, desde diciembre de 2011 hasta agosto de 2017 provenientes de la ciudad de Concepcion, Chile. Se recopilaron datos demográficos, clínicos y variables poligráficas. Estadística descriptiva, expresando resultados en mediana y rango. Se determinó asociación entre índice de apnea hipopnea (IAH) y saturación mediante Rho de Spearman; considerando significancia p < 0,05. Resultados: Se analizaron 190 estudios. Edad 7,9 años $(1,0-20,6)$, varones $61 \%$. Diagnósticos: enfermedades neuromusculares (ENM) (24,2\%), daño pulmonar crónico $(21,1 \%)$, obstrucción de vía aérea superior (OVAS) (19,5\%), daño neurológico (11\%), síndrome de Down (8,9\%) malformaciones VAS (7,4\%), hipoventilación central (3,7\%), obesidad $(2,6 \%)$ y otros $(1,6 \%)$. El $55,3 \%$ de las PG resultaron alteradas; síndrome de apnea hipopnea obstructiva del sueño (SAHOS) leve en $53,3 \%$, moderado $30,5 \%$ y severo $16,2 \%$. No se observaron diferencias significativas en IAH entre grupos de patologías $(p=0,032)$, destacando un mayor IAH en Obesidad 9,0 (0,41-51) y ENM 23,9 (0,4-36,6). Se constató asociación entre IAH y parámetros de saturación; saturación promedio ( $\mathrm{rho}=-0,425 ; \mathrm{p}=0,001)$; mínima (rho $=-0,654 ; \mathrm{p}=0,001$ ); $\mathrm{y}$ saturación bajo $90 \%(r h o=0,323 ; p=0,001)$ en la totalidad de la muestra. Discusión: Existió un alto porcentaje de SAHOS en pacientes pediátricos de riesgo, en especial en aquellos con ENM y obesidad. La PG es una herramienta accesible e implementable en un hospital público; situación potencialmente extrapolable a otros centros asistenciales.
\end{abstract}

Palabras clave: Poligrafía; Apnea; Trastornos Respiratorios del Sueño; Estudios de sueño 


\section{Abstract}

The early diagnosis of Sleep Disordered Breathing (SDB) may allow proper intervention. Currently, polygraphy (PG) is a reliable and accessible alternative. Objective: To describe and analyze the PG of children $\geq 1$ year old with suspicion of SDB. Patients and Method: PG of children $\geq 1$ year old and adolescents from Concepcion, Chile, with suspected SDB were included, from December 2011 to August 2017. Demographic, clinical and polygraphic variables were collected. It was used descriptive statistics, expressing results in median and range. The association between apnea-hypopnea index (AHI) and oxygen saturation was determined by Spearman's Rho, considering significance of $\mathrm{p}<0.05$. Results: 190 studies were analyzed. Age 7.9 years old (1.0-20.6), 61\% males. Diagnosis: neuromuscular disease (NMD) (24.2\%), chronic lung damage (21.1\%), upper airway obstruction (UAO) (19.5\%), neurological damage (11\%), Down syndrome (8.9\%), upper airway malformations $(7.4 \%)$, central hypoventilation $(3.7 \%)$, obesity (2.6\%), and others (1.6\%). 55.3\% were altered PG, with $53.3 \%$ of mild Obstructive Sleep Apnea Hypopnea Syndrome (OSAHS), 30.5\% moderate, and 16.2\% severe. There were no significant differences in AHI between groups of pathologies $(p=0.032)$, highlighting a higher AHI in obese patients 9 (0.41-51), and those with NMD 23.9 (0.4-36.6). It was found association between $\mathrm{AHI}$ and oxygen saturation parameters: mean saturation (rho $=-0.425 ; \mathrm{p}=0.001)$, minimum ( $r$ o $=-0.654 ; \mathrm{p}=0.001)$, and oxygen saturation below $90 \%(\mathrm{rho}=0.323 ; \mathrm{p}=0.001)$ in the whole sample. Discussion: There was a high percentage of OSAHS in at-risk pediatric patients, especially in those with NMD and obesity. PG is an accessible and implementable tool in a public hospital, a situation that can potentially be extrapolated to other healthcare centers.

\section{Keywords:}

Polygraphy;

Apnea;

Sleep Disordered

Breathing;

Sleep Studies

\section{Introducción}

Los trastornos respiratorios del sueño (TRS) incluyen un amplio espectro de entidades, que van desde el roncador primario, la resistencia de la vía aérea superior y el síndrome de apnea hipopnea obstructiva del sueño (SAHOS) en sus distintos grados (leve, moderado y severo) $)^{1,2}$. Metaanálisis epidemiológicos internacionales dan cuenta de un 7,5\% de roncadores habituales; $y$ de una prevalencia del SAHOS infantil en rango entre $1-4 \% \%^{2,3}$.

Existen algunas patologías en niños y adolescentes, donde la frecuencia de los TRS en mucho mayor, por lo tanto, los esfuerzos diagnósticos deber considerarse en su enfrentamiento. Dentro de estos se incluyen: hipertrofia adenotonsilar, obesidad, enfermedades genéticas, enfermedades neuromusculares (ENM), parálisis cerebral y malformaciones craneofaciales ${ }^{4}$.

Los TRS, potencialmente pueden generar repercusiones multisistémicas que incluyen alteraciones neurocognitivas, cardiovasculares y metabólicas; la cuales, se relacionan con el grado de severidad y se objetivan mediante un estudio de sueño $0^{5,6}$.

El examen de elección para el diagnóstico de los TRS es la polisomnografía (PSG); no obstante, su disponibilidad limitada y alto costo, emergen alternativas como la poligrafía (PG), que es más accesible, de menor costo y sus resultados pueden ser más representativos al patrón respiratorio habitual de un niño, pues puede realizarse en domicilio ${ }^{4}$. La PG posee un alto índice de concordancia con respecto a la PSG para el estudio de los TRS; ha sido utilizada y recomendada en distintos grupos de pacientes de riesgo ${ }^{7-10}$. Guías internacionales de reciente publicación consideran que la PG es un examen la gran utilidad en población pediátrica y es la principal alternativa a la $\mathrm{PSG}^{11}$.

Un diagnóstico adecuado y una intervención oportuna, podrían permitir evitar o disminuir las potenciales consecuencias de los TRS, especialmente de índole neurocognitiva; adicionalmente pueden disminuir su impacto en calidad de vida y costo en salud ${ }^{12,13}$.

El objetivo de este estudio fue describir y analizar las PG realizadas a niños mayores de 1 año de edad con sospecha de TRS controlados en un hospital público de nuestro país.

\section{Pacientes y Método}

\section{Diseño}

Estudio restrospectivo que incluyó registros de PG realizadas a niños y adolescentes mayores a 1 año de edad, con sospecha de TRS derivados al Policlínico de Sueño del Servicio de Pediatría, Hospital Guillermo Grant Benavente de Concepción, en el período comprendido entre diciembre 2011 a agosto 2017. Distintos especialistas derivaron a pacientes en riesgo de SAHOS, según anamnesis a padres y tutores legales sobre el sueño (Anexo 1).

Se recopilaron datos demográficos, clínicos y variables poligráficas, considerando la duración total del estudio, duración del estudio validado, índice de apnea 


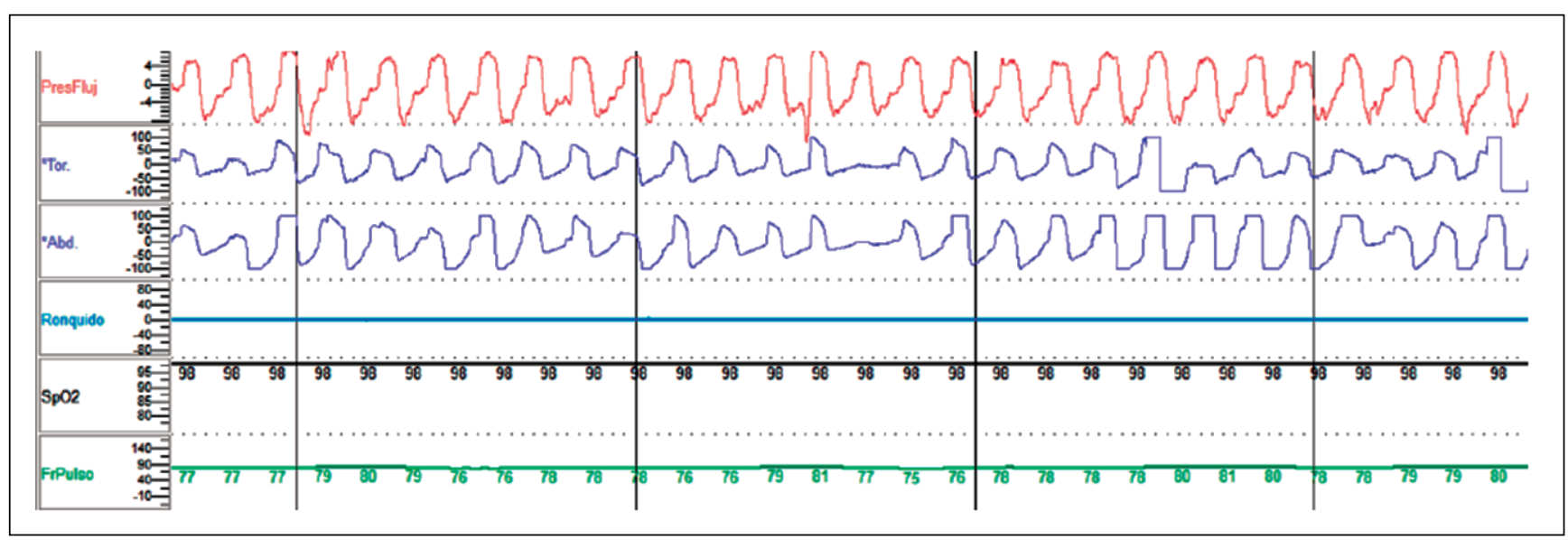

Figura 1. Registro poligráfico normal. En orden descendente se muestran canales evaluados; flujo nasal con transductor de presión, banda torácica, banda abdominal, micrófono, saturación de oxígeno y frecuencia cardiaca.

hipopnea (IAH), índice de apnea hipopnea obstructiva y mixta (IAHOM), índice de apneas centrales (IAC), saturación mínima, saturación promedio y porcentaje de saturación bajo el $90 \%$ durante el estudio. Se excluyeron los pacientes menores de 1 año de edad y aquellos de usuarios de oxigenoterapia o ventilación mecánica durante el examen. En aquellos pacientes que contaban con más de una poligrafía sólo se consideró el primer estudio.

\section{Poligrafía}

Para la realización de PG se utilizó el equipo Alice Pdx (Philips Respironics); que incluyó el registro de los siguientes canales; flujo nasal con transductor nasal de presión, saturación de oxígeno, frecuencia cardiaca, micrófono y banda torácica y abdominal; el cual fue instalado por un profesional capacitado sobre aspectos técnicos y metodológicos del examen (figura 1).

Se consideraron como criterios de aceptabilidad aquellas con al menos 4 horas de registro, con menos del $20 \%$ del tiempo de registro ocupado por desconexiones y/o artefactos. Se excluyeron los registros que no cumplían con estas condiciones. La Severidad del SAHOS fue categorizada de acuerdo al valor del IAH en normal (IAH < 1), leve (IAH 1-5), moderado (IAH $5-10)$ y severo $(\mathrm{IAH}>10)^{5,6}$.

\section{Análisis estadístico}

Los resultados de las variables con distribución normal se expresaron en promedio y desviación estándar, mientras que los datos sin distribución normal se expresaron en mediana y rango. Se empleó análisis de varianza o el test de Kruskal Wallis para comparar los parámetros poligráficos de exámenes alterados entre patologías. Adicionalmente, se determinó la correlación entre IAH y variables de saturación mediante el cálculo del coeficiente Rho de Spearman. Finalmente se realizaron correlaciones parciales determinando como variable de confusión el tipo de patología. El análisis estadístico se realizó en el paquete estadístico SPSS stadistics v23, definiendo un valor de $\mathrm{p}<0,05$ como significativo.

\section{Resultados}

Durante el período estudiado se realizaron 366 PG. Se excluyeron 154 (42\%) por uso de oxigenoterapia, ventilación mecánica y examen repetido. De las 212 poligrafías restantes, se excluyeron 22 casos (10,37\%) por registros no interpretables de los cuales 14 fue por tiempo insuficiente, 7 por pérdida de sensor de flujo y 1 por pérdida de sensor de oximetría. Finalmente quedaron 190 poligrafías para el análisis (figura 2).

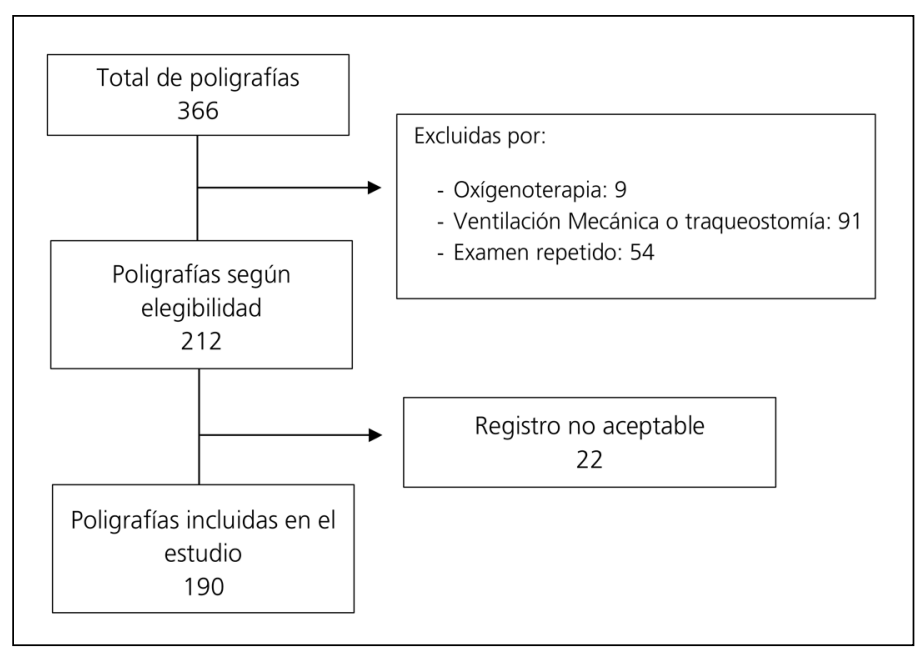

Figura 2. Flujograma criterios de elegibilidad. 
La mediana de edad de la muestra fue 7,8 años $(1,0$ $20,6)$ y el $61 \%(\mathrm{n}=116)$ de sexo masculino. Respecto a los diagnósticos de los pacientes, $24,2 \%$ presentaron ENM, $21 \%$ daño pulmonar crónico y $19,5 \%$ a obstrucción de la vía aérea superior $(\mathrm{n}=37)$. La tabla 1 presenta los diagnósticos de los pacientes estudiados.

El promedio de duración total de las PG fue 9,1 \pm

\begin{tabular}{|c|c|c|}
\hline Diagnóstico & $\mathrm{n}$ & (\%) \\
\hline Enfermedades neuromusculares & 46 & $(24,2)$ \\
\hline Daño pulmonar crónico & 40 & $(21,1)$ \\
\hline Obstrucción de la vía aérea superior & 37 & $(19,5)$ \\
\hline Daño neurológico & 21 & (11) \\
\hline Síndrome de Down & 17 & $(8,9)$ \\
\hline Malformaciones vía aérea superior & 14 & $(7,4)$ \\
\hline Síndrome de hipoventilación central & 7 & $(3,7)$ \\
\hline Obesidad & 5 & $(2,6)$ \\
\hline Otros & 3 & $(1,6)$ \\
\hline Total & 190 & $100)$ \\
\hline
\end{tabular}

1,9 horas, con un período de tiempo validado de 7,2 $\pm 1,3$ horas. La saturación promedio del examen fue $95,9 \pm 2,6 \%$, con un promedio de saturación mínima de $85,1 \pm 10,9 \%$. La mediana de IAH fue $1,6(0-51)$ y de IAHOM fue 1,5 (0-50) y el IAC $0(0-15,1)$.

El $44,7 \%(n=85)$ de las poligrafías analizadas resultaron normales. De las alteradas, el 53,3\% $(n=56)$ fue clasificado como SAHOS leve, el 30,5\% ( $\mathrm{n}=32)$ moderado y el $16,2 \%(n=17)$ severo. Los resultados de las poligrafías alteradas según categoría diagnóstica se detallan en la tabla 2.

No se encontró diferencia significativa en el IAH entre los grupos de patologías ( $\mathrm{p}=0,032)$, sin embargo, destaca un mayor IAH en los grupos de pacientes obesos 9,0 (0,41 - 51,0); ENM 3,9 (0,4-36,6) y aquellos con daño neurológico $3,7(0,5-13,9)$.

Se estableció correlación entre IAH y saturación promedio (rho $=-0,425 ; \mathrm{p}=0,001$ ), IAH y saturación mínima (rho $=-0,654 ; \mathrm{p}=0,001)$ e IAH y saturación bajo $90 \%(\mathrm{rho}=0,323 ; \mathrm{p}=0,001)$ en la totalidad de la muestra (figura 3 ).

Finalmente, para el análisis de correlaciones parciales, existió correlación entre IAH y saturación promedio (rho $=-0,372 ; p=0,001)$, IAH y saturación mínima $(-$ rho $=0,670 ; p=0,001)$ e IAH y saturación bajo $90 \%($ rho $=0,195 ; \mathrm{p}=0,007)$.

Tabla 2. Resultados poligrafías alteradas según diagnóstico

\begin{tabular}{|c|c|c|c|c|c|c|c|c|c|c|}
\hline Variable & ENM & DPC & OVAS & DN & SD & MVAS & SDHC & $\mathrm{OB}$ & Otros & Valor $p^{a}$ \\
\hline (n) & 46 & 40 & 37 & 21 & 17 & 14 & 7 & 5 & 3 & - \\
\hline $\begin{array}{l}\text { PG alteradas } \\
(n / \%)\end{array}$ & $34 / 73,9$ & $19 / 47,5$ & $20 / 54,05$ & $16 / 76,1$ & $17 / 100$ & $11 / 78,5$ & $3 / 42,8$ & $3 / 60$ & $2 / 66,6$ & - \\
\hline Edad & $\begin{array}{c}11,2 \\
(1,3-16,5)^{*}\end{array}$ & $\begin{array}{c}5,38 \\
(1,5-17)\end{array}$ & $\begin{array}{c}4,2 \\
(2,2-12,5)^{*}\end{array}$ & $\begin{array}{c}7,5 \\
(1-17,5)\end{array}$ & $\begin{array}{c}5,6 \\
(1-12,2)\end{array}$ & $\begin{array}{c}4,9 \\
(1,5-15,8)\end{array}$ & $\begin{array}{c}14,3 \\
(13-15)\end{array}$ & $\begin{array}{c}12 \\
(8-13,5)\end{array}$ & $\begin{array}{c}9,9 \\
(9,6-10,2)\end{array}$ & 0,001 \\
\hline $\begin{array}{l}\text { Tiempo total } \\
\text { validado }(\mathrm{h})\end{array}$ & $\begin{array}{c}7,3 \\
(4-10,5)\end{array}$ & $\begin{array}{c}7,4 \\
(4-9,7)\end{array}$ & $\begin{array}{c}7,5 \\
(6-9)\end{array}$ & $\begin{array}{c}7 \\
(4,8-9,4)\end{array}$ & $\begin{array}{c}7,5 \\
(5-10,3)\end{array}$ & $\begin{array}{c}7,5 \\
(5,2-8,6)\end{array}$ & $\begin{array}{c}7,1 \\
(6,5-7,4)\end{array}$ & $\begin{array}{c}7,5 \\
(6,18,2)\end{array}$ & $\begin{array}{c}6,9 \\
(6,6-7,3)\end{array}$ & 0,858 \\
\hline $\begin{array}{l}\text { Tiempo validado } \\
\text { (min) }\end{array}$ & $\begin{array}{c}441 \\
(243-630)\end{array}$ & $\begin{array}{c}444 \\
(240-582)\end{array}$ & $\begin{array}{c}449 \\
(360-540)\end{array}$ & $\begin{array}{c}422 \\
(288-564)\end{array}$ & $\begin{array}{c}450 \\
(300-618)\end{array}$ & $\begin{array}{c}450 \\
(312-516)\end{array}$ & $\begin{array}{c}426 \\
(390-444)\end{array}$ & $\begin{array}{c}450 \\
(363-492)\end{array}$ & $\begin{array}{c}417 \\
(396-438)\end{array}$ & 0,872 \\
\hline $\begin{array}{l}\text { Saturación pro- } \\
\text { medio }\end{array}$ & $\begin{array}{c}97 \\
(89-98)\end{array}$ & $\begin{array}{c}95 \\
(89-98)\end{array}$ & $\begin{array}{c}97 \\
(93-98)\end{array}$ & $\begin{array}{c}96 \\
(89-98)\end{array}$ & $\begin{array}{c}96 \\
(87-98)\end{array}$ & $\begin{array}{c}96 \\
(94-98)\end{array}$ & $\begin{array}{c}96 \\
(92-96)\end{array}$ & $\begin{array}{c}96 \\
(92-97)\end{array}$ & $\begin{array}{c}85 \\
(80-89)\end{array}$ & 0,065 \\
\hline $\begin{array}{l}\text { Saturación } \\
\text { mínima }\end{array}$ & $\begin{array}{c}87 \\
(0-94)\end{array}$ & $\begin{array}{c}86 \\
(69-93)\end{array}$ & $\begin{array}{c}86 \\
(58-94)\end{array}$ & $\begin{array}{c}85 \\
(69-95)\end{array}$ & $\begin{array}{c}87 \\
(65-94)\end{array}$ & $\begin{array}{c}89 \\
(60-93)\end{array}$ & $\begin{array}{c}87 \\
(73-87)\end{array}$ & $\begin{array}{c}88 \\
(50-90)\end{array}$ & $\begin{array}{c}72 \\
(59-85)\end{array}$ & 0,814 \\
\hline IAH & $\begin{array}{c}3,9 \\
(0,4-36,6)\end{array}$ & $\begin{array}{c}3,2 \\
(0,4-10,3)\end{array}$ & $\begin{array}{c}2,7 \\
(0,3-18,2)\end{array}$ & $\begin{array}{c}3,7 \\
(0,5-13,9)\end{array}$ & $\begin{array}{c}2,7 \\
(0-19,1)\end{array}$ & $\begin{array}{c}1,8 \\
(0,5-20,6)\end{array}$ & $\begin{array}{c}3,4 \\
(1,2-10,5)\end{array}$ & $\begin{array}{c}9 \\
(04,1-51)\end{array}$ & $\begin{array}{c}1,1 \\
(0,3-2)\end{array}$ & 0,032 \\
\hline IAHOM & $\begin{array}{c}3,6 \\
(0,4-24,9)\end{array}$ & $\begin{array}{c}2,6 \\
(0-9)\end{array}$ & $\begin{array}{c}2,2 \\
(0,2-18,2)\end{array}$ & $\begin{array}{c}3,5 \\
(0,5-13,7)\end{array}$ & $\begin{array}{c}2,6 \\
(0-19)\end{array}$ & $\begin{array}{c}1,8 \\
(0,5-20,2)\end{array}$ & $\begin{array}{c}3,4 \\
(1,2-9)\end{array}$ & $\begin{array}{c}9 \\
(4-50)\end{array}$ & $\begin{array}{c}1,1 \\
(0,3-2)\end{array}$ & 0,0372 \\
\hline IAC & $0(0-15,1)$ & $0(0-8,5)$ & $0(0-1)$ & $0(0-4)$ & $0(0-0,4)$ & $0(0-0,4)$ & $0(0-1)$ & $0,1(0-1)$ & $0(0-0)$ & 0,978 \\
\hline
\end{tabular}

ENM: enfermedad neuromuscular; DPC: daño pulmonar crónico; OVAS: obstrucción vía aérea superior; DN: daño neurológico; SD: síndrome de Down; MVAS: malformación vía aérea superior; SDHC: síndrome hipoventilación central; OB: obesidad; PG: poligrafías; IAH: índice de apnea hipopnea; IAHOM: índice de apnea hipopnea obstructiva y mixta; IAC: índice de apneas centrales. ${ }^{2}$ Kruskal Wallis. *Diferencia entre grupo estadísticamente significativa $p<0,05$ ENM y OVAS. 


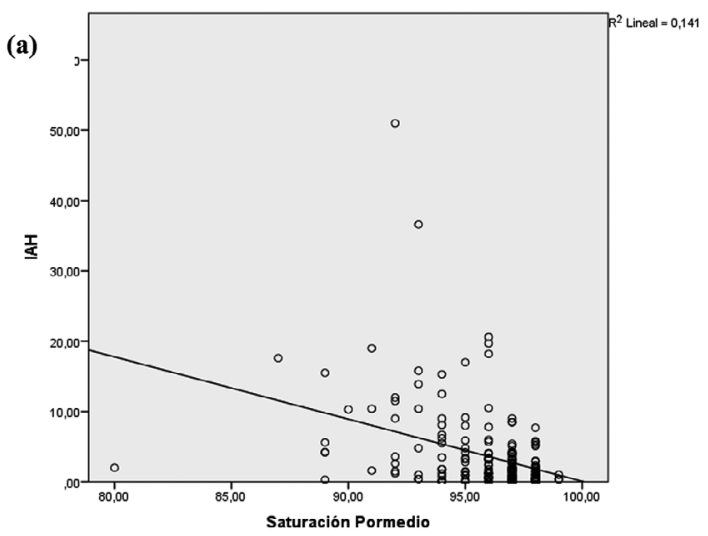

Coeficiente Rho de Spearman; rho=-0,425; $p=0,001$ (b)

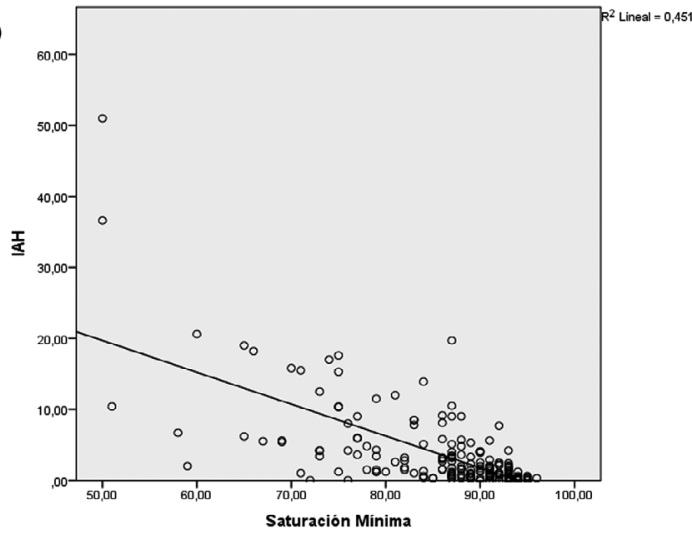

Coeficiente Rho de Spearman; rho=-0,654; $p=0,001$

(c)

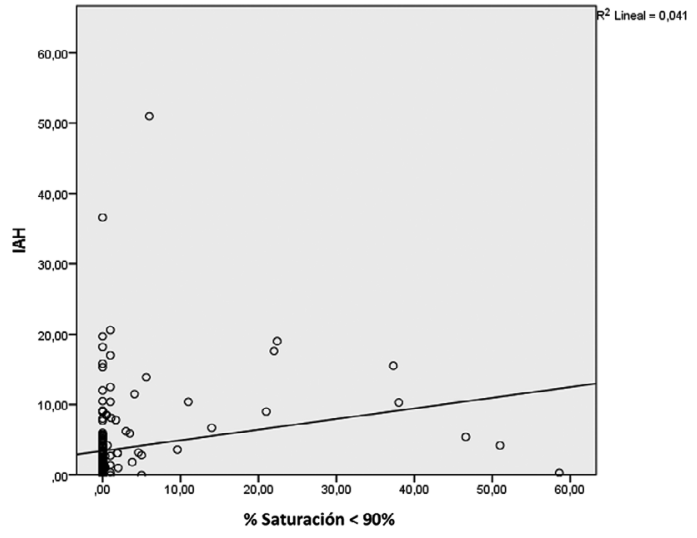

Coeficiente Rho de Spearman; rho=0,323; $p=0,001$

Figura 3. Correlación entre IAH y Saturación Promedio (a), Saturación mínima (b) y \% Desaturación menor a 90\% (c)

\section{Discusión}

El presente estudio buscó mostrar la experiencia con estudios poligráficos en pacientes mayores de un año, con riesgo de TRS, manejados en un hospital público del nivel terciario de nuestro país; considerando la gran variedad de entidades clínicas que requieren una evaluación con este tipo de herramientas diagnósticas para asumir conductas terapéuticas ${ }^{14}$.

Es relevante recalcar que la PSG es el examen de elección para el diagnóstico de los TRS, sin embargo su disponibilidad en nuestro medio es bastante reducido en la actualidad, por lo cual algunas recomendaciones internacionales y de expertos, recomiendan utilizar la PG como examen alternativo, para mejorar la accesibilidad diagnóstica ${ }^{11,15}$.

Mediante este estudio fue posible constatar que el $90 \%$ de los estudios cumplieron con criterios de validez en el primer examen, rendimiento semejante a lo observado en estudios previos tanto en el medio hospitalario como en el domicilio ${ }^{9,16}$. Esto puede interpre- tarse también como que el 10\% de los pacientes, debe repetirse el examen para ser interpretado en forma más confiable. Las principales razones atribuidas a la validez del examen fueron el registro de tiempo insuficiente y la pérdida del sensor de flujo.

Los principales diagnósticos de los pacientes estudiados incluyeron entidades crónicas, como ENM y daño pulmonar crónico; no obstante la mayor prevalencia de TRS en pacientes pediátricos se vincula con hipertrofia adenotonsilar. Esto se explica, pues los menores fueron reclutados principalmente a partir de un policlínico de sueño e indicados por profesionales especialistas, generalmente en escenarios clínicos complejos, siendo este estudio relevante para establecer conductas como el soporte ventilatorio y/o cirugías específicas.

Un 55\% de las PG resultaron alteradas, en distinto grado según los criterios establecidos y sin diferencia significativa entre los índices respiratorios y el tipo de patología; aunque existió una tendencia a mayor alteración en pacientes en Obesos, ENM y DN. Con respecto a la edad de estos pacientes, hubo diferen- 
cias significativas entre dos grupos solamente (ENM y OVAS).

Estudios epidemiológicos refieren que el 1-4\% de los niños presentan SAHOS, sin embargo, este porcentaje aumenta, cuando existe factores de riesgo y/o entidades nosológica como ENM, malformaciones craneofaciales, obesidad, síndromes genéticos, enfermedad pulmonar crónica entre otros ${ }^{2,3,14}$. La población estudiada, posee un alto riesgo de TRS, lo que explica el alto porcentaje de alteración.

En conclusión, existe un alto porcentaje de SAHOS en pacientes pediátricos de riesgo, en especial en aquellos con ENM y obesidad. El 90\% de las PG se consideró interpretable, cifra similar a lo reportado en otras experiencias publicadas. Este estudio sugiere que la PG es una herramienta accesible e implementable, en un hospital público de Chile para el estudio de los TRS, situación potencialmente extrapolable a otros centros asistenciales.

\section{Responsabilidades Éticas}

Protección de personas y animales: Los autores declaran que los procedimientos seguidos se conformaron a las normas éticas del comité de experimentación humana responsable y de acuerdo con la Asociación Médica Mundial y la Declaración de Helsinki.

Confidencialidad de los datos: Los autores declaran que han seguido los protocolos de su centro de trabajo sobre la publicación de datos de pacientes.

Derecho a la privacidad y consentimiento informado: Los autores han obtenido el consentimiento informado de los pacientes y/o sujetos referidos en el artículo. Este documento obra en poder del autor de correspondencia.

\section{Conflicto de intereses}

Los autores declaran no tener conflicto de intereses.

Anexo 1. Flujograma de derivación, ejecución e informe de poligrafías

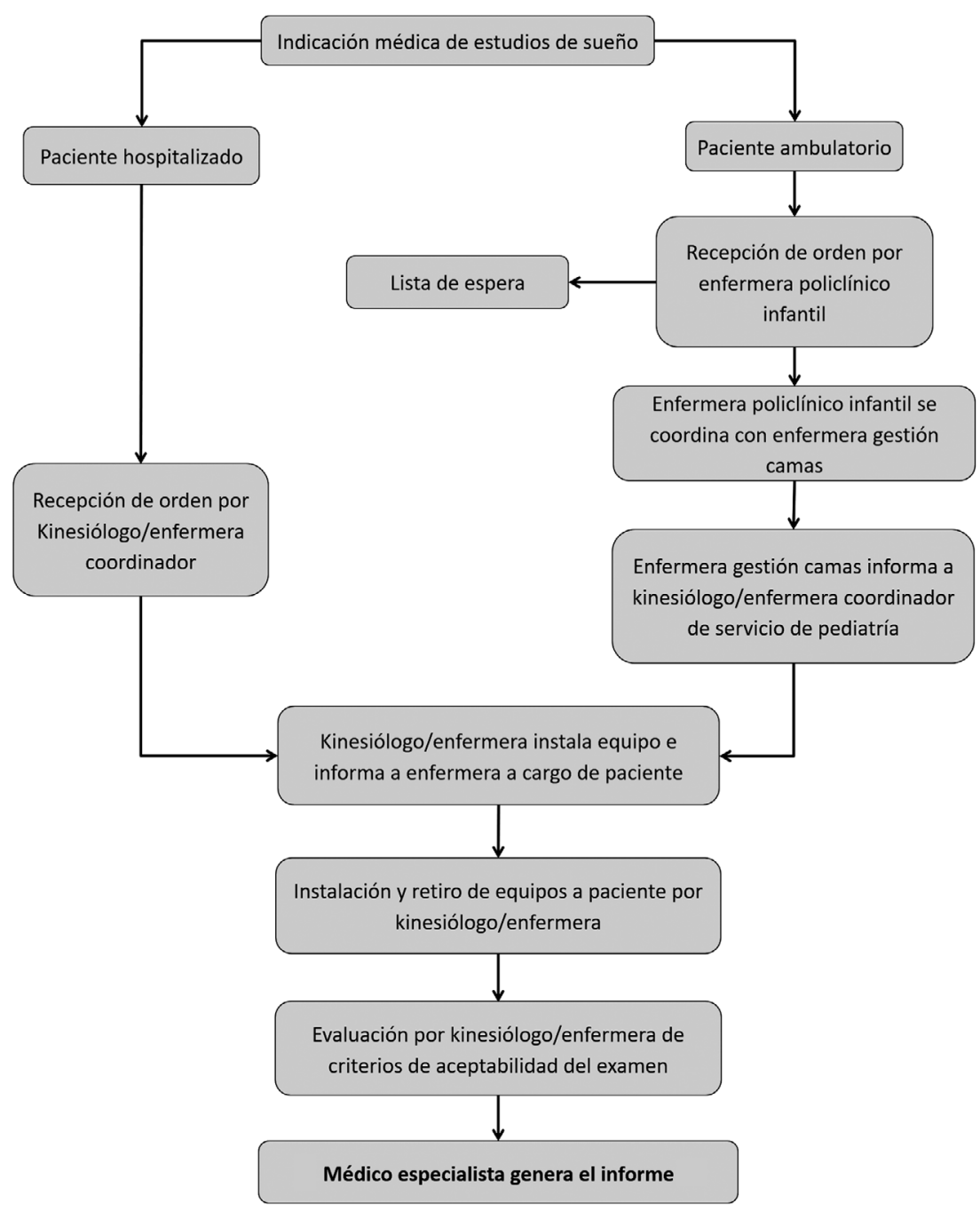




\section{Referencias}

1. Marcus CL, Brooks LJ, Ward SD, Draper K, Gozal D, Halbower AC, et al. Diagnosis and Management of Childhood Obstructive Sleep Apnea Syndrome. Pediatr 2012;130:714-55.

2. Zenteno D, Verbal D, Barraza C, Fuentes C. Epidemiología de los Trastornos Respiratorios del Sueño de Pediatría. Neumol Pediatr 2017;12:49-54.

3. Lumeng JC, Chervin RD. Epidemiology of pediatric obstructive sleep apnea. Proc Am Thorac Soc 2008;5:242-52.

4. Zenteno D, Salinas P, Vera R, Brockmann $\mathrm{P}$, Prado F. Enfoque pediátrico para el estudios de los trastornos respiratorios del sueño. Rev Chil Pediatr 2010;81:445-55.

5. Hunter SJ, Gozal D, Smith DL, Philby MF, Kaylegian J, Kheirandish-Gozal L. Effect of Sleep-disordered Breathing Severity on Cognitive Performance Measures in a Large Community Cohort of Young School-aged Children. Am J Respir Crit Care Med 2016;194:739-47.

6. Berry R, Budhiraja R, Gottlieb D, Gozal D, Iber C, Kapur V, et al. Rules for Scoring Respiratory Events in Sleep:
Update of the2007. AASM Manual for the Scoring of Sleep and Associated

7. Alonso Álvarez ML, Teran Santo SJ, Cordero Guevara JA, Navazo Eguia AI, Ordax Carbajo E, Masa Jiménez JF, et al. Reliability of respiratory polygraphy for the diagnosis of sleep apnea hypopnea syndrome in children. Arch Bronconeumol 2008;44:318-23.

8. Hull J, Aniapravan R, Chan E, Chatwin M, Forton J, Gallagher J, et al. British Thoracic Society guideline for respiratory management of children with neuromuscular weakness. Thorax 2012;67:1-40.

9. Zenteno D, Rodríguez I, Rivas C, Peña R, Molina I, Tapia J. Poligrafía en niños con enfermedad neuromuscular. Rev Chil Enferm Respir 2015;31:152-9.

10. Hill CM, Evans HJ, Elphick H, Farquhar M, Pickering RM, Kingshott R, et al. Prevalence and predictors of obstructive sleep apnoea in young children with Down syndrome. Sleep Med 2016;2728:99-106.

11. Kaditis AG, Alonso Alvarez ML, Boudewyns A, Alexopoulos EI, Ersu $\mathrm{R}$, Joosten $\mathrm{K}$, et al. Obstructive sleep disordered breathing in 2- to 18-year-old children: diagnosis and management. Eur Respir J 2016;47:69-94.

12. Marcus CL, Moore RH, Rosen CL, Giordani B, Garetz SL, Taylor HG, et al. Childhood Adenotonsillectomy Trial (CHAT). A randomized trial of adenotonsillectomy for childhood sleep apnea. N Engl J Med 2013;368:2366-76.

13. Elso MJ, Brockmann P, Zenteno D. Consecuencias del Síndrome de Apnea Obstructiva del Sueño. Rev Chil Pediatr 2013; 84:128-37.

14. Joosten KF, Larramona H, Miano S, Van Waardenburg D, Kaditis AG, Vandenbussche N, et al. How do we recognize the child with OSAS? Pediatr Pulmonol 2017;52:260-71.

15. Gozal D, Kheirandish-Gozal L, Kaditis AG. Home sleep testing for the diagnosis of pediatric obstructive sleep apnea: the times they are a changing...! Curr Opin Pulm Med 2015; 21:563-8.

16. Brockmann P, Pérez JL Moya A. Feasibility of unattended home polysomnography in children with sleep-disordered breathing. Int J Pediatr Otorhinolaryngol 2013;77:1960-4. 